\title{
Agrippa d'Aubigné
}

\section{Hécatombe à Diane}

Édition Julien Goeury.

Saint-Étienne : Publications de l'Université de Saint-Etienne, 2007, 239 p.

L'Hécatombe à Diane n'avait pas fait l'objet d'une nouvelle édition depuis celle de Bernard Gagnebin chez Droz à Genève en 1948. Autant dire l'opportunité du travail de Julien Goeury, qui vient éclairer sous un nouveau jour l'œuvre amoureuse d'Agrippa d'Aubigné. L'Hécatombe ne constitue qu'une partie d'un plus vaste ensemble, communément appelé Le Printemps, recueil de poésie amoureuse figurant pour l'essentiel dans le manuscrit 159 du fonds Tronchin de Genève, que l'auteur révisa mais qu'il ne publia pas de son vivant. Se fondant sur le défrichement préalable de Jean-Raymond Fanlo, grand connaisseur des manuscrits d'Aubigné, Julien Goeury revient dans la première section de son introduction sur la complexité génétique de l'œuvre. Le Printemps regroupe en vérité un ensemble hétéroclite d'œuvres profanes, dont les sonnets de l'Hécatombe, les odes et les stances, ne constitueraient qu'un sous-ensemble intitulé La Jeunesse. Pour rendre compte de la totalité du Printemps, il faudrait ajouter les nombreuses pièces éparses dans le manuscrit 159, d'inspiration profane, mythologique ou amoureuse. Voilà qui complique la tâche du futur éditeur de l'œuvre amoureuse d'Aubigné. Pour les seuls sonnets de l'Hécatombe, l'existence du manuscrit 157, qui propose une nouvelle version, autographe celle-ci, du premier livre de la Jeunesse, rend l'entreprise éditoriale plus aisée. L'examen des états successifs des textes révèle le travail de récriture pointilleuse et de recomposition d'ensemble par l'auteur. L'analyse rigoureuse des manuscrits permet d'établir de surcroît que l'œuvre prétendument de jeunesse fit l'objet de relectures et de corrections constantes jusqu'à la vieillesse, en particulier durant cette période d'activité manuscrite et éditoriale que représente l'exil genevois d'Aubigné, à savoir la dernière décennie de sa vie, 1620-1630. C'est la datation même des pièces amoureuses, qu' il faut donc revoir à la hausse : fin de siècle pour certaines, voire début du suivant pour d'autres. Nous sommes loin de la datation des premiers éditeurs qui situaient la rédaction des sonnets dans les années 70, du temps de l'idylle avec Diane de Salviati. A dire vrai, la composition des sonnets s'échelonnerait sur une cinquantaine d'années. Le poète aime à revenir sur ses textes de même qu'il se plaît à leur donner un prolongement tardif : en témoigne la rédaction des pièces hétéroclites des Petites Euvres meslees, qui couvre un spectre d'années aussi large.

La deuxième section de l'introduction s'attache, pour sa part, à retracer les étapes du roman d'amour de Diane Salviati et du poète avant de conclure sur le rôle structurant de la figure féminine dans l'œuvre et dans l'imaginaire d'Aubigné. 
Nièce de la Cassandre de Ronsard, Diane ne sert pas uniquement les ambitions d'un canzoniere à la française ; elle vient hanter la mémoire du poète au point qu'il continuera, la rupture consommée, à célébrer Diane, après avoir juré pourtant « ne chante $[\mathrm{r}]$ que de Dieu », en déclinant l'amour suivant les modes littéraires. Julien Goeury montre avec conviction le brassage poétique de l'inspiration amoureuse. L'Hécatombe reprend à nouveaux frais la fiction pétrarquiste de l'amant malheureux sur le modèle ronsardien des années 50. A l'heure où triomphe la vogue néo-pétrarquiste avec Desportes, où la manière de Pétrarque est frappée d'obsolescence, Aubigné retrempe la poésie amoureuse aux sources vives du premier pétrarquisme, et ce jusque dans ses relectures et récritures tardives. Son idylle avec Diane lui fournit l'occasion de régler son pas sur celui de Ronsard : la comparaison des amours, on l'aura compris, tient de l'imitation littéraire et donc de la rivalité poétique, si humble soit la posture adoptée. Mais les sonnets n'échappent pas, malgré les protestations de son auteur, à la pression des modes. Aubigné partage l'hésitation des poètes contemporains entre la tentation néo-pétrarquiste et l'autorité ronsardienne : comme Birague, Nuysement, Verville, Aubigné imite Desportes tout en célébrant Ronsard, comme eux il cède à la vogue italienne au détriment de l'inspiration antique. Sauf que le poète ressource les codes et les conventions, auxquels il ne se prête que par jeu ou défi poétiques, au tragique sénéquien, au style mignard ou à l'énergie de la satire qui lui permettent de garder, non sans insolence, son indépendance de poète. On regrette que l'introduction qui analyse brillamment les thèmes de l'œuvre et la position singulière du sujet lyrique, ne s'interroge pas plus longuement sur l'identité poétique du recueil au regard de l'histoire littéraire et sociale de son temps.

Suite à l'introduction, est précisé le « protocole d'édition ». Julien Goeury reproduit le texte du manuscrit 157, modernisé pour les besoins de la collection, avec correction d'erreurs manifestes, rétablissement de mots manquants et d'une ponctuation souvent inexistante. En revanche, la graphie et l'orthographe du manuscrit 157 ont été respectées, à l'exception des majuscules que l'éditeur a conservées pour les noms propres et pour les noms communs qui les comportaient systématiquement. Pour ce qui est de l'annotation, un double système de références permet de séparer variantes des manuscrits et commentaires pour l'essentiel d'ordre lexical, syntaxique et stylistique. D’autre part, les sonnets sont précédés de la préface, assortie d'une notice efficace, aux trois livres de la Jeunesse, telle qu'elle figure dans le manuscrit 159. Bien que la préface ne concerne pas le seul recueil de l'Hécatombe, mais les trois livres de la Jeunesse, peut-être même l'ensemble du Printemps, l'éditeur a fait le choix de la publier à l'ouverture des sonnets, car elle éclaire le projet poétique de l'ensemble de la production amoureuse. 
Le volume s'achève sur des annexes réunissant les sonnets du manuscrit 159 dont la version diverge considérablement de la leçon du manuscrit 157, sur un rappel utile des principes de versification régissant la poésie de l'époque, sur un glossaire, un index, enfin une table des incipit, bref toutes choses qui faciliteront l'accès de l'œuvre aux étudiants à qui s'adresse d'abord cette édition de poche, efficace et ferme, solide et convaincante.

VÉRONIQUE FERRER, Université de Bordeaux

\section{Le Statut de l'écrivain}

Direction scientifique Lise Sabourin. Travaux de Littérature, publiés par l'ADIREL Xx (2007), $518 \mathrm{p}$.

Depuis des années l'Association pour la Diffusion de la Recherche Littéraire (ADIREL) organise des colloques très suivis sous l'impulsion magistrale de sa présidente, Madeleine Bertaud. Les Travaux de Littérature, qui en sont le résultat durable, continuent à paraître régulièrement comme en témoigne le présent volume consacré à l'histoire du statut de l'écrivain, sous la direction de Lise Sabourin, professeur à l'Université Nancy 2.

La nature et la fonction changeantes de l'écrivain au cours de l'histoire fait l'objet de nombreuses études, surtout depuis les déclarations fracassantes lancées par Roland Barthes et Michel Foucault sur « la mort de l'auteur » à la fin des années soixante (R. Barthes, « La Mort de l'auteur, $\gg$ Manteia 5, 1968, repris dans les Euvres complètes, Paris : Seuil, 1994, tome 2; M. Foucault, « Qu'est-ce qu'un auteur? 》 Bulletin de la Société française de philosophie, juillet-septembre 1969, pp. 73-104). Avec le renouveau d'intérêt critique pour la bibliologie, les aspects matériels de la production du livre reçoivent un regain d'attention de la part non seulement des historiens et des sociologues mais encore de tous ceux qui étudient les mouvements littéraires, les modes de transmission des savoirs et les niveaux de notoriété de tout « ouvrier en écriture ».

Après un premier trio de réflexions lexicologiques sur les diverses dénominations de l'écrivain ( $\mathrm{H}$. Walter sur les tentatives de classement, Ph. Ménard sur le vocabulaire spécialisé du Moyen Âge, I. Turcan sur le statut du prosateur de Ménage à Sartre) une trentaine de communications savantes explorent les conditions d'existence du romancier, du dramaturge, du poète et des auteurs de «genres mineurs » au fil de l'histoire, des modes et des fortunes littéraires. La richesse et la diversité des apports est remarquable, même si le Moyen Âge et le $\mathrm{xx}^{\mathrm{e}}$ siècle apparaissent 